\title{
Cardiovascular risk factors in patients with a ortic stenosis predict prevalence of coronary artery disease but not of aortic stenosis: an angiographic pair matched case-control study
}

\author{
J R Ortlepp, F Schmit, T Bozoglu, P Hanrath, R Hoffmann
}

Heart 2003;89:1019-1022

See end of article for authors' affiliations

Correspondence to: Dr Jan R O rtlepp, Medical Clinic I, University Hospital of Aachen, Pauw elsstrasse 30, 52057 Aachen, G ermany;

jrortlepp@ukaachen.de

Accepted 7 April 2003

\begin{abstract}
Background: Traditional cardiovascular risk factors have been associated with aortic stenosis and coronary artery disease. As these two conditions often coexist, the association of cardiovascular risk factors with aortic stenosis may reflect confounding.

Objective: To compare the cardiovascular risk profile in patients with severe aortic stenosis undergoing elective coronary angiography with that of patients without a ortic stenosis or calcification undergoing coronary angiography for suspected coronary artery disease.

Methods: 523 patients referred for elective diagnostic left heart catheterisation because of severe aortic stenosis formed the case population; 3925 patients without valve disease referred for elective diagnostic left heart catheterisation formed the base control population. Of the latter, 523 were pair matched to the case population for sex, age, and prevalence of relevant coronary artery disease, forming a pair matched control population. Cardiovascular risk factors (male sex, hypertension, hypercholesterolaemia, smoking, diabetes mellitus, family history of coronary artery disease) were assessed in all the patients.

Results: N one of the cardiovascular risk factors was more prevalent in patients with aortic stenosis than in the base control population or in the pair matched control population. However, male sex, hypercholesterolaemia, smoking, diabetes mellitus, and a family history of coronary artery disease were significantly associated with the presence of additional coronary artery disease in patients with aortic stenosis.

Conclusions: Cardiovascular risk factors are commonly present in patients with aortic stenosis. However, when compared with controls matched for age, sex, and angiographically defined coronary artery disease, no risk factor was significantly associated with the prevalence of aortic stenosis. Thus other factors are likely to be more important in the pathogenesis of aortic stenosis.
\end{abstract}

G enerally accepted cardiovascular risk factors for coronary artery disease are age, hypertension, hypercholesterolaemia, smoking, and diabetes mellitus. ${ }^{12}$ Calcific aortic stenosis is the most frequent acquired valvar disease and has a serious prognosis when it becomes symptomatic. ${ }^{3}$ Calcific aortic stenosis and coronary artery disease share many similarities. Both increase in prevalence with age, but they are not simply a normal consequence of aging. ${ }^{45}$

Calcific aortic stenosis is associated with the same risk factors as coronary artery disease. ${ }^{4}{ }^{67}$ Furthermore aortic sclerosis is itself associated with a $50 \%$ increased risk of cardiovascular mortality and myocardial infarction. ${ }^{8}$ The large cardiovascular health study (CHS) showed that raised serum cholesterol is associated with the presence of aortic valve disease. ${ }^{4}$ Recent smaller studies have provided evidence of the importance of high concentrations of low density lipoprotein (LDL) cholesterol for the development of aortic stenosis, ${ }^{9}$ and lowering LDL cholesterol with statins might be an option to retard the progression of this disease. ${ }^{10}$ However, despite their similarities the two diseases are likely to have a different pathogenesis.

Aortic stenosis and coronary artery disease often co-exist, so an association of cardiovascular risk factors with aortic stenosis might be confounded by this association. Moreover, influences other than cardiovascular risk factors-genetic ${ }^{11} 12$ or inflammatory, ${ }^{11}{ }^{13}$ for example-could trigger the pathogenesis of aortic stenosis. M ost earlier studies ${ }^{4-7} 9$ have lacked coronary angiographic data in control patients and thus the prevalence of coincidental coronary artery disease may have been underestimated.

Our hypothesis in this study was that cardiovascular risk factors may be associated with aortic stenosis because of their confounding association with coronary artery disease. We therefore compared the cardiovascular risk profile in patients with severe aortic stenosis undergoing elective diagnostic coronary angiography with that in patients without aortic stenosis or calcification who were undergoing elective diagnostic coronary angiography for suspected coronary artery disease.

\section{METHODS}

Study population

Within an area of approximately 300000 inhabitants, the study centre is the only reference centre undertaking coronary angiography. Consecutive patients with calcific aortic stenosis formed the case population. Inclusion criteria were first elective diagnostic coronary angiography and an invasively determined mean gradient across the aortic valve of $>40 \mathrm{~mm} \mathrm{Hg}$. Exclusion criteria were mild or moderate aortic stenosis, severe aortic regurgitation, or other relevant valvar disease. In all, 523 patients fulfilled these criteria and formed the case population.

Consecutive patients with clinically suspected coronary artery disease referred for coronary angiography formed the control population. Inclusion criteria were first elective diagnostic coronary angiography and a normal aortic valve on fluoroscopy. Exclusion criteria were known valvar disease, calcification of the aortic valve on fluoroscopy, and a gradient of 
Table 1 Traditional cardiovascular risk factors in patients with severe aortic stenosis and in controls with normal aortic valves (not matched)

\begin{tabular}{|c|c|c|c|c|}
\hline & Severe aortic stenosis & Normal aortic valve & p Value & Odds ratio $(95 \% \mathrm{Cl})$ \\
\hline$n$ & 523 & 3925 & & \\
\hline Age (years) (mean (SD)) & $70(8)$ & $61(11)$ & $<0.001$ & \\
\hline Clear coronaries or coronary sclerosis & $246(47 \%)$ & $1426(36 \%)$ & & \\
\hline $\begin{array}{l}\text { Stenosis of }>50 \% \text { in at least one coronary vessel } \\
\text { Cardiovascular risk factors }\end{array}$ & $277(53 \%)$ & $2499(64 \%)$ & & \\
\hline Male sex & $287(55 \%)$ & $2642(67 \%)$ & 0.001 & $0.59(0.49$ to 0.71$)$ \\
\hline Hypertension & $308(59 \%)$ & $2282(58 \%)$ & 0.777 & $1.03(0.86$ to 1.24$)$ \\
\hline Hypercholesterolaemia & $258(49 \%)$ & $2623(67 \%)$ & 0.001 & $0.49(0.40$ to 0.58$)$ \\
\hline Smoking & $213(41 \%)$ & $1807(46 \%)$ & 0.022 & $0.81(0.67$ to 0.97$)$ \\
\hline $\mathrm{FH}$ of coronary artery disease & $144(28 \%)$ & $1289(33 \%)$ & 0.015 & $0.78(0.63$ to 0.95$)$ \\
\hline Diabetes mellitus & $106(20 \%)$ & $699(18 \%)$ & 0.183 & $1.17(0.93$ to 1.47$)$ \\
\hline
\end{tabular}

Values are $n(\%)$ unless stated.

$\mathrm{Cl}$, confidence interval; $\mathrm{FH}$, family history.

more than $5 \mathrm{~mm} \mathrm{Hg}$ across the aortic valve during left heart catheterisation. In all, 3925 patients fulfilled these criteria. From this control population, 523 patients were pair matched for age, sex, and the presence of coronary artery disease and formed the matched control population.

All patients gave their written informed consent. The study was approved by the local ethics committee of the University Hospital of Aachen.

\section{Left heart catheterisation}

Coronary angiography, including at least four views of each coronary artery, was carried out in all patients. Coronary angiograms were classified as showing either no significant coronary artery disease- that is, clear coronary arteries or coronary sclerosis (signs of atherosclerosis with stenosis less than $50 \%$ - or definite coronary artery disease (stenosis greater than $50 \%$ in at least one vessel). The gradient across the aortic valve was measured by pull back of the catheter from the left ventricle into the ascending aorta. The mean gradient was cal culated by a computer assisted program (M etek, Roetgen, Germany).

\section{Cardiovascular risk factors}

Cardiovascular risk factors were as follows:

- hypercholesterolaemia: serum cholesterol > $5.2 \mathrm{mmol} / \mathrm{l}$ ( $200 \mathrm{mg} / \mathrm{dl}$ ) or medically treated
- arterial hypertension: blood pressure $>140 / 90 \mathrm{~mm} \mathrm{Hg}$ or medically treated

- diabetes mellitus: overnight fasting serum glucose $>7.0 \mathrm{mmol} / \mathrm{l}(126 \mathrm{mg} / \mathrm{dl})$ on at least two occasions or medically treated

- smoking: regular smoking habit

- positive family history of coronary artery disease: a first degree relative with known coronary artery disease at any age.

\section{Data collection}

The cardiovascular risk profile was assessed by physicians unaware of the study, and data were added to the computer record of the individual patient before angiography was done. Angiograms were interpreted by two experienced physicians.

\section{Statistical analysis}

Continuous data are presented as mean (SD), and qualitative data as frequencies. Differences between frequencies of cardiovascular risk factors were assessed by cross tabulation $\chi^{2}$ analysis. For estimation of chance, the odds ratios for single cardiovascular risk factors were calculated by $\chi^{2}$ analysis. Calculations were done using SPSS for windows, version 10.0. Significant differences were defined as a probability value of $p<0.05$.

Table 2 Traditional cardiovascular risk factors in patients with severe aortic stenosis and controls with normal aortic valves (pair matched for age, sex, and the presence of stenotic coronary artery disease on angiography)

\begin{tabular}{|c|c|c|c|c|}
\hline & Severe aortic stenosis without CAD & Normal aortic valve without CAD & $\mathrm{p}$ Value & O dds ratio $(95 \% \mathrm{Cl})$ \\
\hline $\mathrm{n}$ & 246 & 246 & & \\
\hline Male sex* & $98(40 \%)$ & $98(40 \%)$ & & \\
\hline Age (years)* (mean (SD)) & $69(8)$ & $69(8)$ & & \\
\hline \multicolumn{5}{|l|}{ Cardiovascular risk factors } \\
\hline Hypertension & $136(55 \%)$ & $154(63 \%)$ & 0.119 & $0.74(0.52$ to 1.06$)$ \\
\hline Hypercholesterolaemia & $94(40 \%)$ & $142(58 \%)$ & 0.001 & $0.45(0.32$ to 0.65$)$ \\
\hline Smoking & $80(33 \%)$ & $61(25 \%)$ & 0.072 & $1.46(0.98$ to 2.16$)$ \\
\hline FH of CAD & $52(21 \%)$ & $60(24 \%)$ & 0.452 & $0.83(0.55$ to 1.27$)$ \\
\hline \multirow[t]{2}{*}{ Diabetes mellitus } & $39(16 \%)$ & $39(16 \%)$ & 1.000 & $1.00(0.62$ to 1.62$)$ \\
\hline & Severe aortic stenosis with CAD & $\mathrm{N}$ ormal a ortic valve with $\mathrm{CAD}$ & & \\
\hline$n$ & 277 & 277 & & \\
\hline Male sex* & $189(68 \%)$ & $189(68 *)$ & & \\
\hline Age (years)* (mean (SD)) & $70(7)$ & $70(7)$ & & \\
\hline \multicolumn{5}{|l|}{ Cardiovascular risk factors } \\
\hline Hypertension & $172(62 \%)$ & $174(63 \%)$ & 0.930 & $0.97(0.69$ to 1.36$)$ \\
\hline Hypercholesterola emia & $164(59 \%)$ & $167(60 \%)$ & 0.862 & $0.96(0.68$ to 1.34$)$ \\
\hline Smoking & $133(48 \%)$ & $117(42 \%)$ & 0.200 & $1.26(0.90$ to 1.76$)$ \\
\hline $\mathrm{FH}$ of CAD & $92(33 \%)$ & $65(24 \%)$ & 0.014 & 1.62 (1.12 to 2.36$)$ \\
\hline Diabetes mellitus & $67(24 \%)$ & $56(20 \%)$ & 0.307 & $1.26(0.84$ to 1.88$)$ \\
\hline
\end{tabular}


Table 3 Traditional cardiovascular risk factors in patients with severe aortic stenosis with and without coronary artery disease

\begin{tabular}{lllll}
\hline & $\begin{array}{l}\text { Severe aortic stenosis } \\
\text { without CAD }\end{array}$ & $\begin{array}{l}\text { Severe aortic } \\
\text { stenosis with CAD }\end{array}$ & p Value & $\begin{array}{l}\text { Odds ratio } \\
(95 \% \text { Cl) }\end{array}$ \\
\hline$n$ & 246 & 277 & & \\
$\begin{array}{l}\text { Age (years) (mean (SD)) } \\
\text { Cardiovascular risk factors }\end{array}$ & $69(8)$ & $70(7)$ & & \\
M ale sex & $98(40 \%)$ & $189(68 \%)$ & $<0.001$ & $3.24(2.26$ to 4.65$)$ \\
Hypertension & $136(55 \%)$ & $172(62 \%)$ & 0.131 & $1.32(0.93$ to 1.88$)$ \\
Hypercholesterolaemia & $94(40 \%)$ & $164(59 \%)$ & $<0.001$ & $2.35(1.65$ to 3.34$)$ \\
Smoking & $80(33 \%)$ & $133(48 \%)$ & $<0.001$ & $1.92(1.34$ to 2.74$)$ \\
FH of CAD & $52(21 \%)$ & $92(33 \%)$ & 0.002 & $1.86(1.25$ to 2.76$)$ \\
Diabetes mellitus & $39(16 \%)$ & $67(24 \%)$ & 0.022 & $1.69(1.09$ to 2.63$)$ \\
\hline
\end{tabular}

\section{RESULTS}

Cardiovascular risk factors of unmatched case and control populations

As shown in table 1, the patients referred for coronary angiography because of severe aortic stenosis were significantly older than those referred for coronary angiography for isolated coronary artery disease. M ale sex, hypercholesterolaemia, smoking, and a family history of coronary artery disease were less often present in patients with aortic stenosis than in the those with normal aortic valves in the unmatched control population.

\section{Cardiovascular risk factors of case and pair matched} control populations

Comparing the cardiovascular risk profile of patients with severe aortic stenosis and pair matched controls (matched for sex, age, and the presence of coronary artery disease), patients with severe aortic stenosis but without relevant coronary artery disease less often had hypercholesterolaemia ( $p=0.001$ ) (table 2 , upper part). In patients with severe aortic stenosis and coronary artery disease, the cardiovascular risk profile was similar to the controls apart from a slightly higher prevalence of a positive family history of coronary artery disease $(p=0.014)$ (table 2 , lower part).

Cardiovascular risk factors and prevalence of coronary artery disease in patients with severe aortic stenosis Male sex, hypercholesterolaemia, smoking, diabetes mellitus, and a family history of coronary artery disease were significantly associated with the presence of coronary artery disease in patients with severe aortic stenosis (table 3 ). There

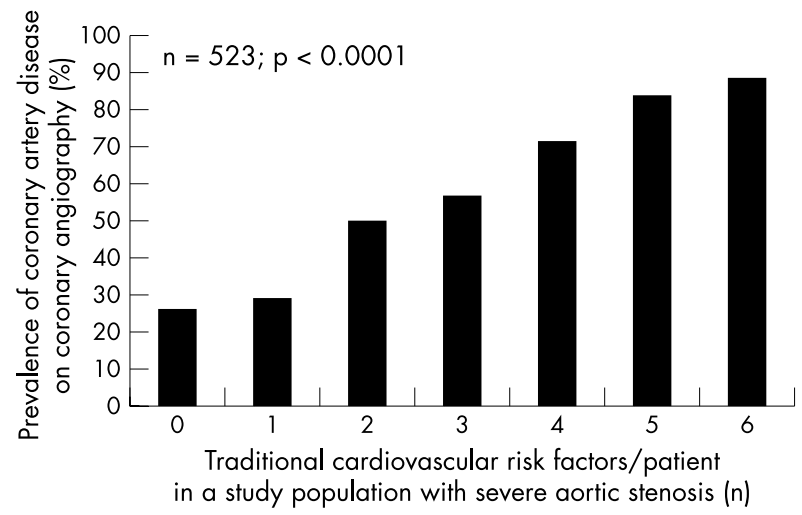

Figure 1 Relation between total number of traditional cardiovascular risk factors per patient (male sex, hypertension, hypercholesterolaemia, smoking, diabetes mellitus, and family history of coronary artery disease) and the prevalence of stenotic coronary artery disease on angiography (diameter stenosis $>50 \%$ in at least one vessel). was only a trend for hypertension to be more frequent in patients with coronary artery disease $(p=0.131)$. As shown in fig 1 , traditional cardiovascular risk factors had an additive effect, with the lowest prevalence of coronary artery disease in patients without these risk factors and the highest prevalence in patients with all six cardiovascular risk factors ( $p<0.0001)$.

\section{DISCUSSION}

Male sex, hypertension, hypercholesterolaemia, smoking, diabetes mellitus, and a family history of the condition are established risk factors for coronary artery disease. ${ }^{12}$ A large echocardiographic study showed that the risk factors associated with the prevalence of aortic sclerosis and aortic stenosis were similar. ${ }^{4}$ However, because of the strong linkage between aortic stenosis and coronary artery disease, ${ }^{8}$ these risk factors may not be related to calcification of the aortic valve but rather to atherosclerosis of the coronary arteries. It is possible that cardiovascular risk factors are associated with aortic stenosis purely because of an association between aortic stenosis and coronary artery disease. Thus, when defining risk factors for aortic stenosis, it is essential to control for atherosclerosis of the coronary arteries.

Our major finding in this study was that none of the traditional cardiovascular risk factors was more prevalent in patients with severe aortic stenosis than in controls. However, cardiovascular risk factors predicted the presence of coronary artery disease in patients with severe aortic stenosis. This finding may indicate that traditional cardiovascular risk factors are associated with aortic stenosis purely on the basis of its link with coronary artery disease.

In this study population there was a lower prevalence of hypercholesterolaemia in patients with aortic stenosis than in those without aortic stenosis. This is of particular interest because there is evidence for an association between serum cholesterol concentrations and calcification of the aortic valve. ${ }^{910}$ Despite the fact that most patients with hypercholesterolaemia do not develop aortic stenosis, cholesterol may be of special importance for calcification in that valve. However, the simple presence of this cardiovascular risk factor does not seem to be a reliable predictor of severe aortic stenosis. Other mechanisms might be important for deposition of cholesterol in the aortic valve. For example, a case report in 1993 described a family with immunological abnormalities and severe aortic val ve cal cification. ${ }^{11}$ Furthermore it is known that stenotic aortic val ves contain infiltrates of macrophages and $T$ lymphocytes as well as an accumulation of plasma lipoproteins. ${ }^{14}{ }^{15}$ Genetic factors and inflammation may be key factors in the initiation and progression of aortic stenosis.

Our study cannot fully prove that cardiovascular risk factors are not involved in the pathogenesis of aortic stenosis, but the study showed that many patients with the same burden of cardiovascular risk factors as patients with aortic stenosis 
have normal aortic valves, whereas the association of cardiovascular risk factors with coronary artery disease remains strong, and in patients with severe aortic stenosis cardiovascular risk factors are highly predictive of the presence of coronary artery disease.

We conclude that further studies are necessary to clarify the pathogenesis of aortic stenosis. Assessment of cardiovascular risk factors al one is not sufficient to identify the mechanisms of valve calcification, and further factors need to be considered.

Authors' affiliations

J R Ortlepp, F Schmitz, T Bozoglu, P Hanrath, R Hoffmann, M edical Clinic I, University Hospital of Aachen, G ermany

\section{REFERENCES}

1 Kannel WB, Dawber TR, Kagan A, et al. Factors of risk in the development of coronary heart disease - six year follow up experience: the Framingham study. Ann Intern Med 1961:55:33-50.

2 Kannel WB, W ilson PW. An update on coronary risk factors. M ed Clin N orth Am 1995;79:951-71.

3 Ross J, Braunwald E. Aortic stenosis. Circulation 1968;38(suppl 1): $61-7$

4 Stewart BF, Siscovick D, Lind BK, et al. Clinical factors associated with calcific aortic valve disease. J Am Coll Cardiol 1997;29:630-4.

5 Lindroos M, Kupari M, Heikkila J, et al. Prevalence of aortic valve abnormalities in the elderly: an echocardiographic study of a random population sample. J Am Coll Cardiol 1993;21:1220-5.
6 Boon A, Cheriex E, Lodder I, et al. Cardiac valve calcification: characteristics of patients with calcification of the mitral annulus or aortic valve. Heart 1997;78:472-4.

7 Aronow WS, Schwart KS, Koenigsberg M. Correlation of serum lipids, calcium, and phosphorus, diabetes mellitus and history of systemic hypertension with presence or absence of calcified or thickened aortic cusps or root in elderly patients. Am / Cardiol 1987:59:998-9.

8 Otto CM, Lind BK, Kitzman DW, et al. Association of aortic-valve sclerosis with cardiovascular mortality and morbidity in the elderly. $N$ Engl I Med 1999:341:142-7.

9 Pohle K, M äffert R, Ropers D, et al. Progression of aortic valve calcification. Association with coronary atherosclerosis and cardiovascular risk factors. Circulation 2001;104:1927-32.

10 Novaro GM, Tiong IY, Pearce G L, et al. Effect of hydroxymethylglutaryl coenzyme $A$ reductase inhibitors on the progression of calcific aortic stenosis. Circulation 2001;104:2205-9.

11 Tentolouris C, Kontozoglou T, Toutouzas P. Familial calcification of aorta and calcific valve disease associated with immunologic abnormalities. Am Heart J 1993;126:904-9.

12 Ortlepp JR, Hoffmann R, O hme F, et al. The vitamin D receptor genotype predisposes the development of calcific a ortic valve stenosis. Heart 2001:85:635-8.

13 Wang $A Y, W 00 \mathrm{~J}, W$ ang $M$, et al. Association of inflammation and malnutrition with cardiac valve calcification in continuous ambulatory peritoneal dialysis patients. I Am Soc N ephrol 2001;12:1927-36.

14 O'Brien KD, Reichenbach DD, M arcovina SM, et al. A polipoprotein B, (a) and $E$ accumulate in the morphological early lesion of "degenerative" valvular a ortic stenosis. Arterioscler Thromb Vasc Biol 1996;16:523-32.

15 Olsson $\mathrm{M}$, Thyberg J, $\mathrm{N}$ ilsson J. Presence of oxidized low density lipoprotein in nonrheumatic stenotic aortic valves. Arterioscler Thromb Vasc Biol 1999;19:1218-22.

\section{IM AGES IN CARDIO LO G Y}

\section{Synergistic anticoagulation and transoesophageal echocardiographic surveillance for persistent left atrial appendage thrombus}

A 49 year old woman was referred for consideration of percutaneous mitral valve commissurotomy caused by symptoms of worsening exertional breathlessness. She had a long history of rheumatic mitral stenosis complicated 10 years previously by a left parietal lobe embolic stroke and the onset of permanent atrial fibrillation. She was anticoagulated with warfarin following this but was left with some residual disability.

Transoesophageal echocardiography (TOE) demonstrated severe mitral stenosis with a large thrombus visible in the left atrial appendage (below left). Anticoagulation control before this had been good (international normalised ratio (INR) 2.5). The patient was offered mitral valve surgery but declined. She was initially treated with clopidogrel $75 \mathrm{mg}$ daily and the target INR increased to 3.5. Repeat TOE at both one and two

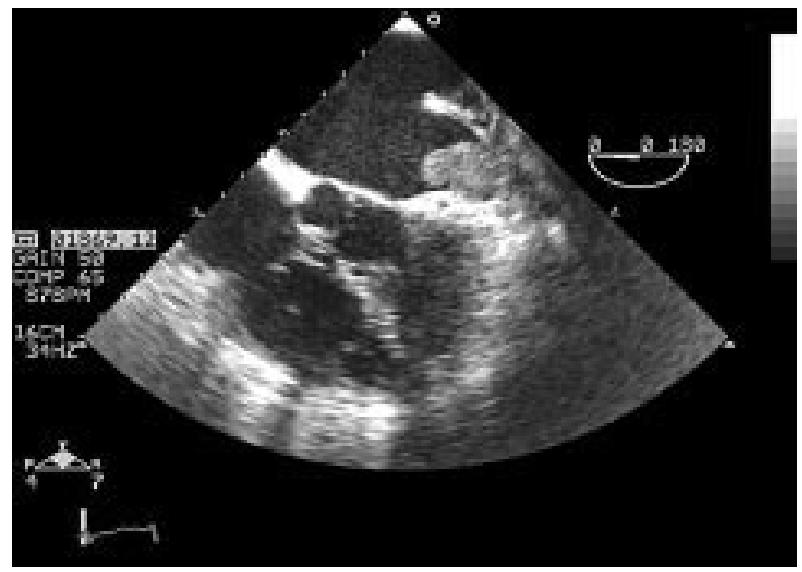

months demonstrated persistence of the thrombus. Aspirin $75 \mathrm{mg}$ was added synergistically to her anticoagulation therapy and serial TOE scanning over the subsequent six months demonstrated a progressive reduction in size (below right).

Although resolution of large left atrial appendage thrombi has been previously reported, this case demonstrates the need to assess the response of large thrombi to anticoagulation by repeated TOE. This case also displays the potential for synergistic antiplatelet treatment in the management of persistent left appendage thrombi.

A R J Mitchell J Timperley A P Banning mitcharj@doctors.org.uk

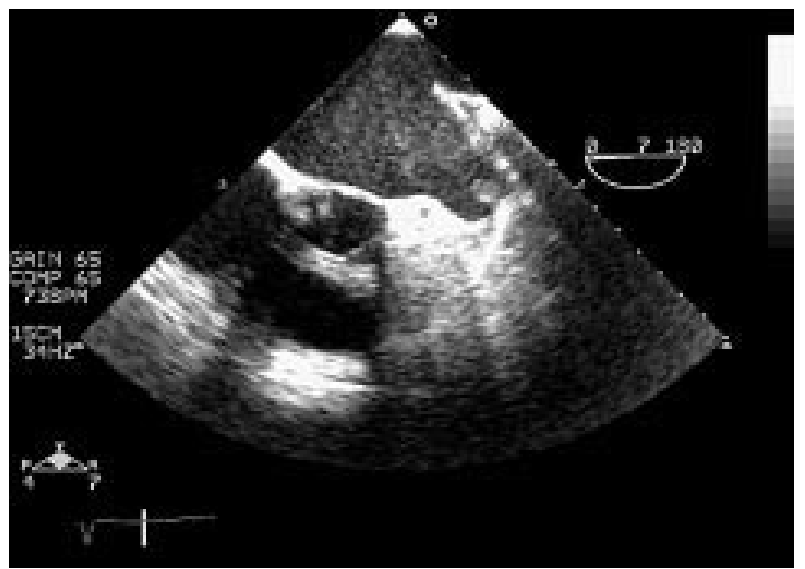

